\title{
Telomerase-specific Type 5 Adenovirus OBP-301
}

National Cancer Institute

\section{Source}

National Cancer Institute. Telomerase-specific Type 5 Adenovirus OBP-301. NCI

Thesaurus. Code C119617.

A replication-competent oncolytic, telomerase-specific adenovirus serotype 5 (Ad5), with potential antineoplastic activity. OBP-301 contains the human telomerase reverse transcriptase (hTERT) gene promoter sequence that drives the expression of the E1A and E1B genes, and is linked to an internal ribosomal entry site (IRES). Upon administration, OBP-301 selectively infects and replicates in cancer cells that are expressing telomerase, which causes cell lysis. This adenovirus does not infect or replicate in normal, healthy cells. OBP-301 may also potentially be used as a chemosensitizer. hTERT, which encodes for the catalytic protein subunit of telomerase, is overexpressed in a variety of cancer cell types but not in normal, healthy cells. The insertion of an IRES further improves selectivity towards telomerase-expressing cancer cells. 